\title{
Benefit of Granulocyte Colony-Stimulating Factor-Primed Donor Lymphocyte Infusion using Cryopreserved Cells for Patients with Acute Leukemia Who Relapsed Late after Hematopoietic Cell Transplantation
}

\author{
Lee $\mathrm{YJ}^{1,2}$, Moon $\mathrm{JH}^{1,2}$ and Sohn SK ${ }^{1,2 *}$ \\ ${ }^{1}$ Division of Hematology-Oncology and Internal Medicine, \\ Kyungpook National University Hospital, Korea \\ ${ }^{2}$ School of Medicine, Kyungpook National University, \\ Korea \\ *Corresponding author: Sohn SK, Division of \\ Hematology-Oncology, Department of Internal Medicine, \\ Kyungpook National University Hospital, 130 Dongduk- \\ Ro, Jung-Gu, Daegu, Korea
}

Received: January 03, 2019; Accepted: February 11, 2019; Published: February 18, 2019

\begin{abstract}
There is no standard therapy for relapse of acute leukemia after allogeneic Hematopoietic Cell Transplantation (allo-HCT). This study evaluated the efficacy of Granulocyte Colony-Stimulating Factor (G-CSF)-Primed Donor Lymphocyte Infusion (DLI) for patients with acute leukemia who relapsed after allo-HCT. We retrospectively reviewed 255 patients who received allo-HCT for acute leukemia/myelodysplastic syndrome. They were divided into two groups based on the $\mathrm{CD} 34^{+}$cell dose they received; patients in the lower $\mathrm{CD} 34^{+}$group received less than $6 \times 10^{6} \mathrm{cells} / \mathrm{kg}$ and those in the higher group received over $6 \times 10^{6}$ cells $/ \mathrm{kg}$. No significant differences were noted between the groups with respect to overall survival, relapse-free survival, and Graft-Versus-Host Disease (GVHD)-free/relapse-free survival. Among the 93 patients with relapse after allo-HCT, 39 patients received G-CSF-primed DLI. These 93 patients were classified into early or late relapse groups as defined by the median time to relapse. In the late relapse group, the one-year overall survival was significantly higher in the DLI group than in the non-DLI group $(53.4 \pm 7.4 \%$ vs. $26.7 \pm 7.4 \%$, $p=0.039$ ), whereas there were no differences in the early relapse group. In addition, the incidence of DLI-induced GVHD did not differ between the two groups. In conclusion, treatment with G-CSF-primed DLI after allo-HCT with a limited $\mathrm{CD} 34^{+}$cell dose is a feasible and effective option, which may replace a second HCT in late relapse patients.
\end{abstract}

Keywords: Donor lymphocyte infusion; Hematopoietic stem cell transplantation; Acute leukemia; Recurrence; Granulocyte colony-stimulating factor

\section{Abbreviations}

allo-HCT: allogeneic Hematopoietic Cell Trangrsplantation; G-CSF: Granulocyte Colony-Stimulating Factor; DLI: Donor Lymphocyte Infusion; GVHD: Graft-Versus-Host Disease; GRFS: Graft-free/Relapse-Free Survival; GVL: Graft-Versus-Leukemia; OS: Overall Survival; AML: Acute Myelogenous Leukemia; MDS: Myelodysplastic Syndrome; ALL: Acute Lymphoblastic Leukemia; KNUH: Kyungpook National University Hospital; aGVHD: acute GVHD; cGVHD: chronic GVHD; RFS: Relapse-Free Survival; PRS: Post-Relapse Survival; PBSCT: Peripheral Blood Stem Cell Transplant; $\mathrm{Bu}$ : Busulfan; Cy: Cytoxan; Flu: Fludarabine; GM-CSF: Granulocyte Macrophage Colony-Stimulating Factor; MTX: Methotrexate; CyA: Cyclosporine A; Tac: Tacrolimus; NRM: Non-Relapse Mortality; HR: Hazard Ratio

\section{Introduction}

Allogeneic Hematopoietic Cell Transplantation (allo-HCT) is a potentially curative therapy for acute leukemia [1]. However, patients with acute leukemia who relapse after allo-HCT show poor prognosis with a median survival of 3-4 months [2]. Second allo-HCT resulted only in 10\%-35\% long-term survival rate, with higher treatment- related mortality. Currently, there is no standard treatment approach for these patients $[3,4]$.

Induction of Graft-Versus-Leukemia (GVL) effects with Donor Lymphocyte Infusions (DLIs) is an attractive option for patients with relapsed hematological malignancies; however, GVL efficacy depends on disease subtype and tumor burden at the time of DLI $[3,5]$. Schmid et al. demonstrated an Overall Survival (OS) benefit of DLI for patients with Acute Myelogenous Leukemia (AML) who relapsed after allo-HCT $(20 \% \pm 3 \%$ vs. $9 \% \pm 2 \%, \mathrm{p}<0.001)$ [6]. Many experts have investigated different strategies to improve patient outcomes, such as dose-escalation of DLIs, addition of immunosuppressive agents to prevent Graft-Versus-Host Disease (GVHD), modified DLI treatment with granulocyte Colony-Stimulating Factor (G-CSF), or pre-DLI chemotherapy for cytoreduction [7-10]. Our institution reported a pilot study on the role of cytarabine with G-CSF-primed DLIs using cryopreserved cells for patients with hematological malignancies who relapsed after allo-HCT [11]. This study aimed to determine the effectiveness of cytarabine combined with G-CSFprimed DLI for patients with acute leukemia who relapsed after alloHCT. This strategy would not only maximize donor convenience but also cost-effectiveness.
Ann Hematol Oncol - Volume 6 Issue 3 - 2019 ISSN : 2375-7965 | www.austinpublishinggroup.com Sohn et al. (C) All rights are reserved
Citation: Lee YJ, Moon JH and Sohn SK. Benefit of Granulocyte Colony-Stimulating Factor-Primed Donor Lymphocyte Infusion using Cryopreserved Cells for Patients with Acute Leukemia Who Relapsed Late after Hematopoietic Cell Transplantation. Ann Hematol Oncol. 2019; 6(3): 1236. 

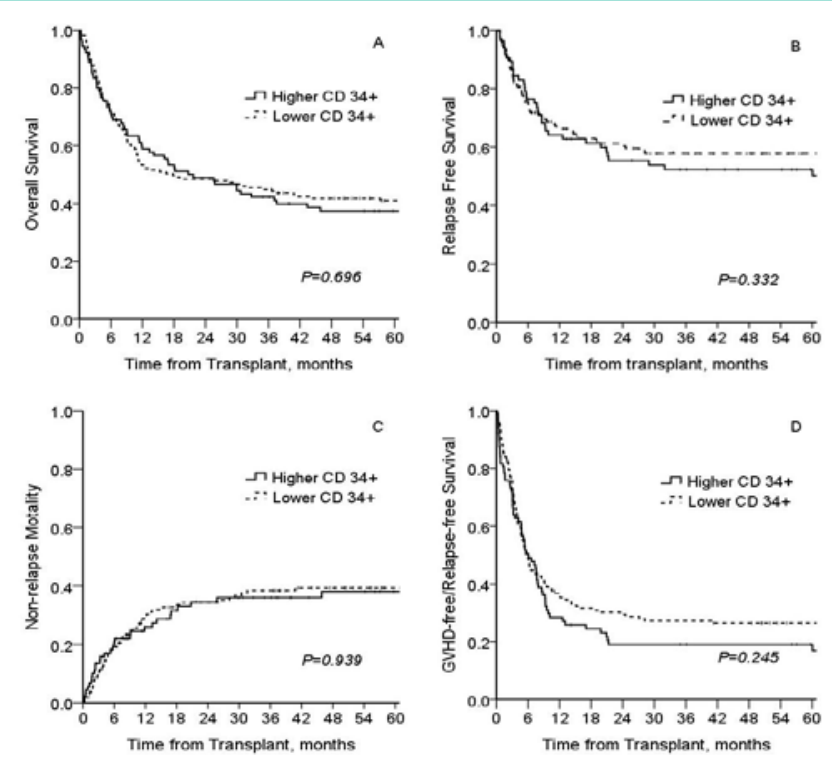

Figure 1: Survival curves according to infused $\mathrm{CD}_{3} 4^{+}$cell dose: Patients were divided into lower or higher $\mathrm{CD} 34^{+}$dose group based on cut-off dose $\left(6 \times 10^{6} / \mathrm{kg}\right)$ of CD $34^{+}$cells. (A) OS: 1 -year OS rates were $53.3 \% \pm 3.9 \%$ and $58.9 \% \pm 5.2 \%$ for the lower and higher groups, respectively $(p=0.696)$. (B) RFS: 1 -year RFS were $66.7 \% \pm 4.0 \%$ and $63.2 \% \pm 5.5 \%$ for the lower and higher groups, respectively $(\mathrm{p}=0.332)$. (C) NRM: 1 -year NRM were $29.5 \% \pm 3.8 \%$ and $25.6 \% \pm 4.7 \%$ for the lower and higher groups, respectively $(p=0.939)$. (D) GRFS: 1 -year GRFS were $35.3 \% \pm 4.0 \%$ and $28.4 \% \pm 5.1 \%$ for the lower and higher groups, respectively $(p=0.332)$.

\section{Materials and Methods}

\section{Data Collection}

We conducted a retrospective review of the medical records of 255 patients who received allo-HCT for AML, Myelodysplastic Syndrome (MDS), or Acute Lymphoblastic Leukemia (ALL) between December 1998 and August 2013 at the Department of Hematology/ Oncology, Kyungpook National University Hospital (KNUH). Clinical and laboratory data were collected from electronic medical records following approval by the KNUH institutional review board.

\section{Definitions}

The risk status at transplantation was based on previously published classification schemes [12]. Poor-risk cytogenetics were classified according to the revised Medical Research Council classification system for AML and the International Prognostic Scoring System for MDS $[13,14]$. Poor-risk cytogenetics for ALL were defined as MLL rearrangement, BCR/ABL1 translocation, hypoploidy, or complex karyotype. Graft failure was defined as the lack of myeloid engraftment in patients surviving in remission for at least 28 days after transplantation. The Keystone staging system was used to score acute GVHD (aGVHD) and chronic GVHD (cGVHD) $[15,16]$. Relapse was defined as the reappearance of leukemic cells in the peripheral blood, bone marrow, or extramedullary lesions after allo-HCT.

A novel composite end-point of refined GVHD-Free/RelapseFree Survival (GRFS) was also examined, where events included grade III-IV aGVHD, systemic therapy requiring cGVHD, relapse, or death [17]. OS was calculated from the date of the first allo-HCT
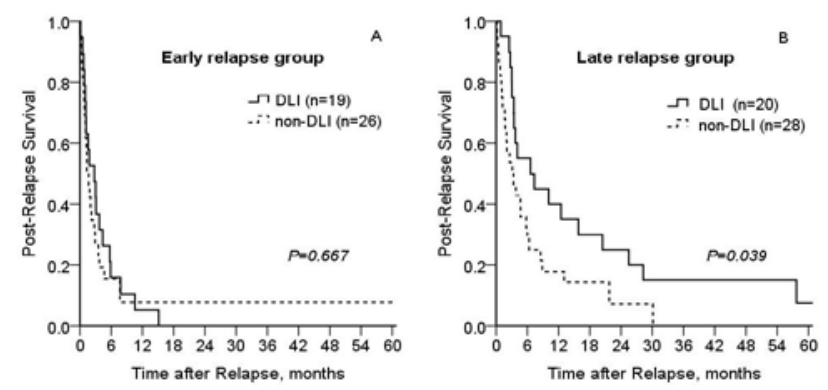

Figure 2: Post-Relapse Survival (PRS) according to the use of DLI: Early relapse and late relapse groups were determined based on median posttransplantation remission duration. (A) In the early relapse group, 1-year PRS rates were $7.7 \% \pm 5.3 \%$ and $5.3 \% \pm 4.3 \%$ for DLI and non-DLI groups, respectively $(\mathrm{p}=0.667)$. (B) In the late relapse group, 1-year PRS rates were $40.0 \% \pm 7.4 \%$ and $17.9 \% \pm 7.2 \%$ for DLI and non-DLI groups, respectively $(p=0.039)$.

to the date of death, or to the last follow-up. Relapse-Free Survival (RFS) was calculated from the date of the first allo-HCT to the date of disease recurrence or to the date of death due to the disease. PostRelapse Survival (PRS) was defined as the time from relapse posttransplantation to death or to the last follow-up [18].

\section{Transplantation procedures}

Preparative regimens for allogeneic Peripheral Blood Stem Cell Transplantation (PBSCT) included busulfan (Bu, 4mg/kg $\mathrm{PO}$ or $0.8 \mathrm{mg} / \mathrm{kg}$ IV for 4 days) and cytoxan (Cy, $60 \mathrm{mg} / \mathrm{kg}$ for 2 days) administered to 100 patients; $\mathrm{Bu}$ (3.2mg/kg for 2-4 days) and fludarabine (Flu, $30 \mathrm{mg} / \mathrm{m}^{2}$ for 6 days) administered to 135 patients; and total body irradiation and Cy $(60 \mathrm{mg} / \mathrm{kg}$ for 2 days) administered to 20 patients. PBSCs were mobilized with $10 \mu \mathrm{g} / \mathrm{kg}$ per day G-CSF (filgrastim (Leukokine); CJ, Co., Korea or lenograstim (Neutrogin'); Chugai Co. Ltd, Tokyo, Japan) alone ( $\mathrm{n}=183,71.8 \%)$ or in combination with a concurrent regimen of $5 \mu \mathrm{g} / \mathrm{kg}$ per day G-CSF and $5 \mu \mathrm{g} / \mathrm{kg}$ per day Granulocyte Macrophage Colony-Stimulating Factor (GM-CSF) ( $n=72,28.2 \%)$ from the donor. Administration of G-CSF and/or GM-CSF was continued, and apheresis was repeated every morning until the targeted number of cells $\left(6 \times 10^{6} / \mathrm{kg} \mathrm{CD} 34+\right.$ cells) was obtained. GVHD prophylaxis consisted of Methotrexate (MTX) and Cyclosporine A (CyA) or MTX and Tacrolimus (Tac).

\section{Collection and infusion of donor lymphocytes}

Collecting the targeted number of PBSCs (more than $6 \times 10^{6} /$ $\mathrm{kg} \mathrm{CD} 34^{+}$cells) allowed us to cryopreserve some PBSCs, including several $\mathrm{CD}^{+}$cells at the time of harvest for transplantation. The extra harvested cells were cryopreserved with dimethylsulfoxide in a nitrogen tank. For those patients who relapsed after allo-HCT, DLI was promptly performed using cryopreserved cells. The CD3+ cellcount was determined by flow cytometry and used to calculate the DLI dose. Before DLI, immunosuppressive agents were discontinued and patients received pre-DLI chemotherapy with high-dose cytarabine $\left(2 \mathrm{~g} / \mathrm{m}^{2}\right.$ twice a day on days 1,3 , and 5$)$. The chimerism status, which was assessed by determining the variable number of tandem repeats or short tandem repeats was compared before and after DLI. All patients underwent a bone marrow examination within 60 days after DLI to assess the response, or sooner if clinically indicated. 
Table 1: Patient characteristics according to $\mathrm{CD} 34^{+}$cell dose.

\begin{tabular}{|c|c|c|c|}
\hline & $\begin{array}{l}\text { Lower CD34 }{ }^{+} \text {dose } \\
\quad(<6 \times 106 / \mathrm{kg})\end{array}$ & $\begin{array}{c}\text { Higher CD34+ } \\
\text { dose } \\
(\geq 6 \times 106 / \mathrm{kg})\end{array}$ & $p$-value \\
\hline Number of patients & $165(64.7)$ & $90(35.3)$ & \\
\hline Median age, years (range) & $39(15-68)$ & $38(16-62)$ & 0.908 \\
\hline Sex (male/female) & $67(40.6) / 98(59.4)$ & $44(48.9) / 46(51.1)$ & 0.273 \\
\hline ECOG PS & & & 0.102 \\
\hline 0 & $57(34.5)$ & $43(47.8)$ & \\
\hline 1 & $106(64.2)$ & $47(52.2)$ & \\
\hline \multicolumn{4}{|l|}{ Disease subtype or Diagnosis } \\
\hline AML & $93(56.4)$ & $56(62.2)$ & 0.072 \\
\hline MDS & $18(10.9)$ & $13(14.4)$ & \\
\hline ALL & $54(32.7)$ & $21(23.3)$ & \\
\hline Poor- risk cytogenetics & $35(23.0)$ & $13(16.7)$ & 0.161 \\
\hline \multicolumn{4}{|l|}{$\begin{array}{l}\text { Disease status at } \\
\text { transplantation }\end{array}$} \\
\hline CR1 & $97(58.8)$ & $46(51.1)$ & \\
\hline Further CR & $14(8.5)$ & $11(12.2)$ & \\
\hline Persistent disease & $54(32.7)$ & $33(36.7)$ & \\
\hline Risk status at transplantation & & 0.512 & \\
\hline Standard risk & $89(53.9)$ & $44(48.9)$ & \\
\hline High risk & $76(46.1)$ & $46(51.1)$ & \\
\hline $\begin{array}{l}\text { Female donor to male } \\
\text { recipient }\end{array}$ & $42(25.5)$ & $6(6.7 \%)$ & 0.022 \\
\hline CMV status & & & 0.23 \\
\hline Donor +/Recipient + & $55(33.3)$ & $35(38.9)$ & \\
\hline Donor +/Recipient - & $17(10.3)$ & $9(10.0)$ & \\
\hline Donor -/Recipient + & $42(25.5)$ & $13(14.4)$ & \\
\hline Donor -/Recipient - & $51(30.9)$ & $33(36.7)$ & \\
\hline Conditioning intensity & & 0.676 & \\
\hline Myeloablative & $113(68.5)$ & $59(65.6)$ & \\
\hline $\begin{array}{l}\text { Reduced intensity } \\
\text { conditioning }\end{array}$ & $52(31.5)$ & $31(34.4)$ & \\
\hline Mobilization & & & 0.246 \\
\hline G-CSF/GM-CSF & $\begin{array}{c}117(70.9) / 48 \\
(29.1)\end{array}$ & $66(73.3) / 24(26.7)$ & \\
\hline $\begin{array}{l}\text { Donor-Recipient HLA } \\
\text { disparity }\end{array}$ & & & 0.535 \\
\hline Matching sibling donor & $96(58.2)$ & $47(52.2)$ & \\
\hline Matching unrelated donor & $34(20.6)$ & $20(22.2)$ & \\
\hline Mismatched related donor & $24(14.5)$ & $19(21.1)$ & \\
\hline Haploidentical-related donor & $11(6.7)$ & $4(4.4)$ & \\
\hline GVHD prophylaxis, & & & 0.242 \\
\hline CsA/MTX & $77(46.7)$ & $49(54.4)$ & \\
\hline Tacrolimus/MTX & $88(53.3)$ & $41(45.6)$ & \\
\hline \multicolumn{4}{|l|}{ In vivo $\mathrm{TCD}$} \\
\hline ATG/alemtuzumab & $73(44.2) / 12(7.3)$ & $32(35.6) / 7(7.8)$ & 0.414 \\
\hline
\end{tabular}

Abbreviations: ECOG PS: Eastern Cooperative Oncology Group Performance Status; AML: Acute Myelogenous Leukemia; MDS: Myelodysplastic Syndrome; ALL: Acute Lymphoblastic Leukemia; CR: Complete Remission; CMV: Cytomegalovirus; G-CSF: Granulocyte Colony-Stimulating Factor; GM-CSF: Granulocyte Macrophage Colony-Stimulating Factor; GVHD: Graft-Versus-Host Disease; Csa: Cyclosporine; MTX: Methotrexate; TCD: T-Cell Depletion; ATG: Anti-Thymoglobulin
Table 2: Transplantation outcomes according to CD $34^{+}$cell dose.

\begin{tabular}{|c|c|c|c|}
\hline & $\begin{array}{l}\text { Lower CD34+ dose } \\
\quad\left(<6 \times 10^{6} / \mathrm{kg}\right)\end{array}$ & $\begin{array}{l}\text { Higher CD34+ dose } \\
\qquad\left(\geq 6 \times 10^{6} / \mathrm{kg}\right)\end{array}$ & $\mathrm{p}$-value \\
\hline Number of patients & $165(64.7)$ & $90(35.3)$ & \\
\hline $\begin{array}{l}\text { Median follow-up, days } \\
\text { (range) }\end{array}$ & $534(8-4962)$ & $636(6-6381)$ & 0.168 \\
\hline $\begin{array}{l}\text { Median stem cell } \\
\text { infusion, (range) }\end{array}$ & & & \\
\hline $\mathrm{CD}_{3} 4^{+} \times 10^{6} / \mathrm{kg}$ & $3.94(0.46-6.00)$ & $7.54(6.01-20.6)$ & $<0.001$ \\
\hline $\mathrm{MNC} \times 10^{8} / \mathrm{kg}$ & $6.91(0.36-12.70)$ & $9.65(3.68-25.12)$ & $<0.001$ \\
\hline $\mathrm{CD}^{+} \times 10^{8} / \mathrm{kg}$ & $2.70(0.05-7.45)$ & $3.10(1.25-10.01)$ & 0.001 \\
\hline \multicolumn{4}{|l|}{ Engraftment } \\
\hline Neutrophil $>500 \mathrm{~mm}^{3}$ & $157(95.2)$ & $84(94.4)$ & 0.81 \\
\hline Platelet $>20,000 / \mathrm{mm}^{3}$ & $150(90.9)$ & $77(85.6)$ & 0.159 \\
\hline \multicolumn{4}{|l|}{$\begin{array}{l}\text { Median time to } \\
\text { engraftment, days }\end{array}$} \\
\hline Neutrophil $>500 / \mathrm{mm}^{3}$ & $13(8-30)$ & $12(9-24)$ & 0.791 \\
\hline Platelet $>20,000 / \mathrm{mm}^{3}$ & $13(8-121)$ & $12(7-161)$ & 0.672 \\
\hline aGVHD & & & 0.594 \\
\hline II-IV & $65(39.4)$ & $39(43.0)$ & \\
\hline III-IV & $16(9.7)$ & $12(13.3)$ & \\
\hline cGVHD, Seattle & & & 0.786 \\
\hline Classic chronic & $44(26.7)$ & $26(28.9)$ & \\
\hline Overlap & $17(10.3)$ & $10(11.1)$ & \\
\hline cGVHD, NIH 2005 & & & 0.821 \\
\hline mild & $36(21.8)$ & $20(22.2)$ & \\
\hline moderate & $30(18.2)$ & $20(22.2)$ & \\
\hline severe & $1(0.6)$ & 0 & \\
\hline Cause of Death & $100(60.1)$ & $58(64.4)$ & 0.712 \\
\hline Relapse & $37(22.4)$ & $24(26.7)$ & \\
\hline Infection & $29(17.6)$ & $12(13.3)$ & \\
\hline GVHD & $18(10.9)$ & $11(12.2)$ & \\
\hline VOD & $10(6.1)$ & $9(10.0)$ & \\
\hline Others & $6(3.6)$ & $2(2.2)$ & \\
\hline
\end{tabular}

Others: 3 patients died of cardiac arrest, 2 patients died of brain hemorrhage, 1 patient died of pulmonary hemorrhage, 1 patient died of malnutrition, 1 patient died of acute renal failure.

Abbreviations: MNC: Mononuclear Cell; aGVHD: acute Graft-Versus-Host Disease; cGVHD: chronic Graft-Versus-Host Disease; VOD: Veno-Occlusive Disease

\section{Statistical analysis}

Categorical data were analyzed using a chi-square test. Survival analysis was conducted using the Kaplan-Meier method, and both groups were compared using a log-rank test. The cumulative incidence of GVHD was calculated using the Gray method considering treatment-related mortality and relapse as competing risks. The Cox proportional regression model was used to analyze potential risk factors affecting survival. Statistical analyses were performed using the SPSS software version 18 (SPSS Inc., Chicago, IL, USA) and EZR (Saitama Medical Center, Jichi Medical University, Saitama, Japan) [19]. 


\section{Results}

\section{Patient and transplant characteristics}

Two hundred and fifty-five patients were analyzed. The median infused cell doses were as follows: mononuclear cell number $7.94 \times 10^{8} /$ $\mathrm{kg}$ (range: $0.36-25.12), \mathrm{CD} 34^{+}$cell number $5.13 \times 10^{6} / \mathrm{kg}$ (range: $0.46-$ 20.6 ), and $\mathrm{CD}^{+}$cell number $2.82 \times 10^{8} / \mathrm{kg}$ (range: $0.05-10.0$ ). Patients were reclassified into two groups according to the targeted $\mathrm{CD} 34^{+}$ cell dose $\left(6 \times 10^{6} / \mathrm{kg}\right)$ based on the KNUH protocol. The lower $\mathrm{CD} 34^{+}$ group $(\mathrm{n}=165,64.7 \%)$ included patients who underwent allo-HCT with CD $34^{+}$cell dose $<6 \times 10^{6} / \mathrm{kg}$, and the higher CD $34^{+}$group $(\mathrm{n}=90$, $35.3 \%)$ included patients who underwent allo-HCT with CD34+ cell dose $\geq 6 \times 10^{6} / \mathrm{kg}$. Patient characteristics are summarized in Table 1 . No statistically significant differences between the two groups were found in the transplantation outcomes, such as the incidence of aGVHD, cGVHD, and relapse rate (Table 2).

\section{Impact of $\mathrm{CD}^{3} 4^{+}$cell dose on GRFS}

The median follow-up duration was 18.1 months, with a range of 0.2 to 209.7 months. The 1-year OS, RFS, and Non-Relapse Mortality (NRM) were $55.3 \% \pm 3.1 \%, 66.0 \% \pm 3.2 \%$, and $28.2 \% \pm 0.3 \%$, respectively. The cumulative incidence of aGVHD and cGVHD was $40.7 \% \pm 0.3 \%$ and $41.6 \% \pm 0.3 \%$, respectively. The unadjusted KaplanMeier estimate of 1 -year GRFS was $32.9 \% \pm 3.1 \%$. No significant difference was found in OS, RFS, NRM, or GRFS between the two groups classified according to the $\mathrm{CD}^{+} 4^{+}$cell dose (Figure 1) Moreover, there was no significant correlation between the number of infused $\mathrm{CD}^{+}$and $\mathrm{CD} 34^{+}$cells (Spearman correlation coefficient: $\mathrm{p}=0.307)$. However, a trend of more $\mathrm{CD}^{+}$cells $\left(>3.1 \times 10^{8} / \mathrm{kg}\right)$ was noted in the higher $\mathrm{CD} 34^{+}$group ( $\mathrm{p}=0.001$, Table 2$)$. In the univariate analysis, patients transplanted with higher $\mathrm{CD} 34^{+}$and $\mathrm{CD} 3^{+}$cell doses did not show an improved GRFS (Table 3, $\mathrm{p}=0.623$ and $\mathrm{p}=0.158$, respectively). The risk status at transplantation was an independent factor associated with worse GRFS (hazard ratio; HR=1.782, 95\% CI: 1.267-2.509, $\mathrm{p}=0.001$, Table 3).

\section{Post-Relapse Survival}

Among the 255 patients, 93 (36.4\%) relapsed after allo-HCT. The median time from allo-HCT to relapse was 4.6 months (range 1.5-59.1). After relapse, 45 patients (48.4\%) were treated with salvage chemotherapy, 9 (9.7\%) with a second allo-HCT, and 39 (41.9\%) with G-CSF-primed DLI. Thereafter, 13 patients (30.0\%) achieved DLI-induced complete remission, 24 progressed, and 2 were not evaluable for response. DLI-induced aGVHD was observed in 24 patients (61.5\%) with a median of 20 days after DLI (range, 3-98 days): ten with grade I, six with grade II, five with grade III, and three with grade IV. As shown in Table 4, univariate analysis revealed that poor-risk cytogenetics $(\mathrm{HR}=2.512, \mathrm{p}=0.015)$, risk status at transplantation $(\mathrm{HR}=4.406, \mathrm{p}<0.001)$, myeloablative conditioning regimen ( $\mathrm{HR}=0.567, \mathrm{p}=0.007), \mathrm{cGVHD}(\mathrm{HR}=0.525, \mathrm{p}=0.006)$, and longer post-transplantation remission duration $(\mathrm{HR}=0.297, \mathrm{p}<0.001)$ were significantly associated with PRS. A longer post-transplantation remission duration was the only independent factor correlated with PRS (HR=0.297, 95\% CI=0.193-0.457, $\mathrm{p}<0.001$ ).

\section{G-CSF-primed DLI effect on PRS}

Among the 39 patients (41.9\%) who received DLIs, 34 received one infusion and five received two infusions. The median number
Table 3: Factors affecting GRFS.

\begin{tabular}{|l|c|c|c|c|c|c|}
\hline & \multicolumn{3}{|c|}{ Univariate } & \multicolumn{3}{c|}{ Multivariate } \\
\hline $\begin{array}{l}\text { CD34+ cell doses, } \\
\text { high }\end{array}$ & 1.2 & $0.822-1.533$ & 0.623 & - & - & - \\
\hline CD3+ median, high & 1.3 & $0.903-1.807$ & 0.158 & - & - & - \\
\hline HCT risk, high & 1.9 & $1.411-2.554$ & $<0.001$ & 1.78 & $1.267-2.509$ & 0.001 \\
\hline $\begin{array}{l}\text { In vivo TCD, yes } \\
\text { Conditioning } \\
\text { intensity }\end{array}$ & 0.92 & $0.675-1.240$ & 0.568 & - & - & - \\
\hline RIC / MAC & 1 & $0.731-1.375$ & 0.986 & - & - & - \\
\hline Donor disparity & & & & - & - & - \\
\hline MUD/MSD & 0.91 & $0.616-1.343$ & 0.633 & - & - & - \\
\hline Haploidentical/MSD & 4.06 & $2.243-7.355$ & $<0.001$ & 4.02 & $1.870-8.658$ & $<0.001$ \\
\hline FD to MR & 0.9 & $0.631-1.269$ & 0.533 & - & - & - \\
\hline Donor CMV positivity & 0.76 & $0.562-1.020$ & 0.066 & - & - & - \\
\hline
\end{tabular}

Abbreviations: GRFS: Graft-Versus-Host Disease-Free; Relapse-Free Survival; HCT: Hematopoietic Cell Transplantation; TCD: T-cell Depletion; RIC: Reduced-Intensity Conditioning; NMA: Non-Myeloablative; MAC: Myeloablative Conditioning; MUD: Matched Unrelated Donor; MSD: Matched Sibling Donor; FD: Female Donor; MR: Male Recipient; CMV: Cytomegalovirus

of $\mathrm{CD}^{+}$cells was $2.82 \times 10^{7} / \mathrm{kg}$ (range: $0.05-10.1$ ). The patient and transplant characteristics according to the post-transplantation remission duration are described in Table 5. The post-transplantation remission duration was divided by the median RFS of 4.6 months (range: 1.5-59.1) to classify early and late relapse groups. For patients with early relapse (remission duration $<4.6$ months), one-year PRS rates were $7.7 \% \pm 5.3 \%$ and $5.3 \% \pm 4.3 \%$ in the DLI and non-DLI groups, respectively (Figure $2 \mathrm{~A}, \mathrm{p}=0.667$ ). For patients with late relapse (remission duration $\geq 4.6$ months), one-year PRS rates were $40.0 \% \pm 7.4 \%$ and $17.9 \% \pm 7.2 \%$ in the DLI and non-DLI groups, respectively (Figure $2 \mathrm{~B}, \mathrm{p}=0.039$ ).

\section{Discussion}

The current study investigated the efficacy of cytarabine-based chemotherapy with G-CSF-primed DLI for patients with acute leukemia who relapsed after allo-HCT. G-CSF-primed DLI treatment after allo-HCT with a limited CD $34^{+}$cell dose $\left(<6 \times 10^{6} / \mathrm{kg}\right)$ is a feasible and effective option in terms of GRFS, donor convenience, and cost. Moreover, this treatment option may replace a second HCT for late relapse patients. Although stem cell dose has already been explored in relation to the incidence of GVHD, relapse, and survival, it remains controversial [20-22]. Preliminary results from our institution demonstrated that transplantation with more than $6 \times 10^{6} / \mathrm{kg} \mathrm{CD} 34^{+}$ cells did not improve refined GRFS (median survival 5.5 months $v s$. 6 months, $\mathrm{p}=0.245$, Figure 1d). Moreover, a higher $\mathrm{CD} 34^{+}$cell dose did not increase the neutrophil or platelet engraftment rate. As the current study found no correlation between the $\mathrm{CD}^{+}$and $\mathrm{CD} 34^{+}$ cell numbers in the harvested cells (Spearman correlation coefficient: $\mathrm{p}=0.307)$, it is planned to limit the $\mathrm{CD} 34^{+}$cell dose $\left(6 \times 10^{6} / \mathrm{kg} \mathrm{CD} 34^{+}\right.$ cells) for transplantation and cryopreserve the rest of the harvested cells for relapse or prophylactic use.

This retrospective study has several limitations, including the heterogeneity of patients and transplant characteristics. Furthermore, DLI treatment has a minimal effect in the case of a rapidly 
Table 4: Factors affecting Post-Relapse Survival (PRS)*

\begin{tabular}{|c|c|c|c|c|c|c|}
\hline & & Univariate & & & Multivariate & \\
\hline & $\mathrm{HR}$ & $95 \% \mathrm{Cl}$ & $p$-value & $\mathrm{HR}$ & $95 \% \mathrm{Cl}$ & $\mathrm{p}$-value \\
\hline Age & 1.16 & $0.753-1.789$ & 0.499 & - & - & - \\
\hline ECOG 0 vs 1 & 1.27 & $0.833-1.945$ & 0.266 & - & - & - \\
\hline CD34+ cell doses ${ }^{* *}$ & 1.16 & $0.764-1.767$ & 0.483 & - & - & - \\
\hline Median $\mathrm{CD}^{+}$cell dose $\mathrm{e}^{*+*+}$ & 0.87 & $0.577-1.303$ & 0.492 & - & - & - \\
\hline Poor- risk cytogenetics & 2.51 & $1.195-5.227$ & 0.015 & - & - & 0.671 \\
\hline Transplantation risk, high & 4.41 & $2.592-6.317$ & $<0.001$ & - & - & 0.192 \\
\hline In vivo TCD, yes & 1.2 & $0.795-1.802$ & 0.389 & - & - & - \\
\hline Myeloablative conditioning regimen & 0.57 & $0.736-0.855$ & 0.007 & - & - & 0.079 \\
\hline Donor disparity & 0.83 & $0.505-1.359$ & 0.455 & - & - & - \\
\hline MMSD vs. MSD & 3.18 & $1.422-7.121$ & 0.005 & - & - & - \\
\hline FD to MR & 1.21 & $0.736-1.981$ & 0.455 & - & - & - \\
\hline Donor CMV positivity & 1.37 & $0.902-2.084$ & 0.14 & - & - & - \\
\hline aGVHD & 0.91 & $0.600-1.374$ & 0.647 & - & - & - \\
\hline cGVHD & 0.53 & $0.333-0.829$ & 0.006 & - & - & 0.14 \\
\hline Delayed PLT engraftment & 1.39 & $0.731-2.623$ & 0.318 & - & - & - \\
\hline Use of DLI & 0.8 & $0.527-1.222$ & 0.306 & - & - & - \\
\hline Median post-transplantation remission duration**** & 0.3 & $0.193-0.457$ & $<0.001$ & 0.57 & $0.375-0.865$ & 0.008 \\
\hline
\end{tabular}

${ }^{*}$ Post-Relapse Survival (PRS) was defined as the time from relapse post-transplantation to death or last follow-up. ** Patients were reclassified into two groups according to the targeted $\mathrm{CD} 34^{+}$cell doses $\left(6 \times 10^{6} / \mathrm{kg}\right)$ by the $\mathrm{KNUH}$ protocol. ${ }^{* * *}$ The median $\mathrm{CD}^{+}$cell dose was $2.82 \times 10^{7} / \mathrm{kg}($ range: $0.05-10.1)$. ${ }^{* * * *}$ Post-transplantation remission duration was divided by the median RFS 4.6 months (range: 1.5-59.1).

Abbreviations: ECOG PS: Eastern Cooperative Oncology Group performance Status; TCD: T-Cell Depletion; MUD: Matched Unrelated Donor; MSD: Matched Sibling Donor; MMSD: Mis-Matched Sibling Donor; FD: Female Donor; MR: Male Recipient; CMV: Cytomegalovirus; aGVHD: acute Graft-Versus-Host Disease; cGVHD: chronic Graft-Versus-Host Disease; PLT: Platelet; DLI: Donor Lymphocyte Infusion

Table 5: Outcomes of DLI treatment according to post-transplantation remission duration.

\begin{tabular}{|l|c|c|c|}
\hline & Early relapse & Late relapse & \\
\hline Number of patients & $19(48.7 \%)$ & $20(51.3 \%)$ & \\
\hline Age, median & $38(19-58)$ & $41(24-56)$ & 0.341 \\
\hline Sex, F/M & $9-$-Oct & $11-$ Sep & 0.634 \\
\hline ECOG PS, 0 vs 1 & $12-J u l$ & $12-$ Aug & 0.839 \\
\hline Diagnosis & & & 0.634 \\
\hline AML & $12(63.2)$ & $14(70.0)$ & \\
\hline MDS & $4(21.1)$ & $3(15.0)$ & \\
\hline ALL & $3(15.8)$ & $3(15.0)$ & \\
\hline Poor-risk cytogenetics & $7(36.8)$ & $7(35.0)$ & 0.841 \\
\hline HCT risk, high & $16(84.2)$ & $9(45.0)$ & 0.011 \\
\hline Purpose of DLI, relapse & $19(100)$ & $20(100)$ & \\
\hline Pre-DLI chemotherapy & $16(84.2)$ & $19(95.0)$ & 0.449 \\
\hline Median CD3 ${ }^{+}$cell $\left(10^{8} / \mathrm{kg}\right)$ & $2.32(1.94-4.66)$ & $2.57(0.11-7.91)$ & \\
\hline Median CD34 ${ }^{+}$cell (10 $\left./ \mathrm{kg}\right)$ & $3.21(1.26-4.85)$ & $3.28(0.98-5.0)$ & \\
\hline Response & & $12(60.0)$ & \\
\hline CR achieved & $5(26.3)$ & 0 & 0.365 \\
\hline Persistent disease & $12(63.2)$ & $140.0)$ & \\
\hline Not available (TRM) & $10(52.6 \%)$ & & \\
\hline DLI induced GVHD & & & \\
\hline
\end{tabular}

Post-transplantation remission duration was divided by the median RFS of 4.6 months (range: 1.5-59.1).

Abbreviations; DLI: Donor Lymphocyte Infusion; F/M; Female/Male; ECOG PS: Eastern Cooperative Oncology Group Performance Status; AML: Acute Myelogenous Leukemia, MDS: Myelodysplastic Syndrome; ALL: Acute Lymphoblastic Leukemia; HCT: Hematopoietic Cell Transplantation; CR: Complete Remission; TRM: Transplant Related Mortality; GVHD: Graft-VersusHost Disease

advancing disease, as evidenced in patients with early relapse who experienced no benefits from DLIs. However, patients with longer post-transplantation remission duration showed better PRS in the DLI group (Figure 2b, 1-year OS $46.7 \% \pm 12.9 \%$, and $21.7 \% \pm 8.9 \%$, $\mathrm{p}=0.039)$.

A second allo-HCT is regarded as an optimal option for patients who relapse after the first transplantation. Yet, this is only available for selected patients due to concerns of high mortality and unavailability of donors. Thus, for the late relapse group, DLI treatment may replace second HCT. A faster recovery can also be expected in the case of chemotherapy followed by G-CSF-primed DLI treatment including a sufficient number of CD $34^{+}$cells. Regarding GRFS, allo-HCT with a limited $\mathrm{CD}^{2} 4^{+}$cell dose $\left(<6 \times 10^{6} / \mathrm{kg}\right)$ is not an inferior therapeutic option to allo-HCT with a higher $\mathrm{CD} 34^{+}$cell dose. Moreover, the surplus cells from the harvest can be cryopreserved at the time of first transplantation. DLI treatment using these cryopreserved cells can then be promptly performed without a new harvest at the time of relapse. From the perspective of donor convenience and costeffectiveness, this strategy is an attractive option for patients with 
dismal prognosis after post-transplantation relapse.

Thus, well-designed prospective clinical trials are required to answer such DLI-related questions as to when, how, and to whom. Previous studies have shown multiple biological effects of G-CSF on peripheral blood stem cells, including the ability to polarize $\mathrm{T}$ cells from Th1 to Th2, promotion of regulatory $\mathrm{T}$ cells, and tolerogenic dendritic cell differentiation [24,25]. In addition, this study found that G-CSF-primed DLI, rather than unstimulated DLI, included more $\mathrm{CD} 34^{+}$cells and led to early recovery. Moreover, interestingly, low mortality was associated with DLI-induced GVHD, and most of the mortality resulted from disease relapse or refractory disease rather than GVHD. In conclusion, G-CSF-primed DLI treatment after allo-HCT with a limited CD34+ cell dose $\left(<6 \times 10^{6} / \mathrm{kg}\right)$ is a feasible and effective option in terms of GRFS, donor convenience, and cost. Moreover, this option may replace second HCT for late relapse patients.

\section{References}

1. Copelan EA. Hematopoietic Stem-Cell Transplantation. NEJM. 2006; 354 1813-1826.

2. Savani BN, Mielke S, Reddy N, Goodman S, Jagasia M, Rezvani K. Management of relapse after allo-SCT for AML and the role of second transplantation. Bone Marrow Transplant. 2009; 44:769-777.

3. Deol A, Lum LG. Role of donor lymphocyte infusions in relapsed hematologica malignancies after stem cell transplantation revisited. Cancer Treat Rev. 2010; 36: 528-538.

4. Barrett AJ, Battiwalla M. Relapse after allogeneic stem cell transplantation Expert Rev Hematol. 2010; 3: 429-441.

5. Chang YJ, Huang XJ. Donor lymphocyte infusions for relapse after allogeneic transplantation. When, if and for whom? Blood Rev. 2013; 27: 55-62.

6. Schmid C, Labopin M, Nagler A, Bornhauser M, Finke J, Volin L, et al. Donor lymphocyte infusion in the treatment of first hematological relapse after allogeneic stem-cell transplantation in adults with acute myeloid leukemia : a retrospective risk ractors analysis and comparison with other strategies by the EBMT Acute Leukemia Working Party. J Clin Oncol. 2007; 25: 4938-4945.

7. Huang XJ, Wang Y, Liu DH, Xu LP, Liu KY, Chen H, et al. Administration of short-term immunosuppressive agents after DLI reduces the incidence of DLI-associated acute GVHD without influencing the GVL effect. Bone Marrow Transplant. 2009; 44: 309-316.

8. Schroeder T, Czibere A, Platzbecker U, Bug G, Uharek L, Luft T, et al. Azacitidine and donor lymphocyte infusions as first salvage therapy for relapse of AML or MDS after allogeneic stem cell transplantation. Leukemia. 2013; 27: 1229-1235.

9. Chen SH, Li X, Huang XJ. Effect of Recombinant Human Granulocyte Colony-Stimulating Factor on T-Lymphocyte Function and the Mechanism of This Effect. Int J Hematol. 2004; 79: 178-184.

10. Huang X, Guo N, Ren H, Zhang Y, Gao Z, Lu D. An improved anti-leukemic effect achieved with donor progenitor cell infusion for relapse patients after allogeneic bone marrow transplantation. Chin Med J (Engl). 2003; 116: 736741.

11. Kim JG, Sohn SK, Kim DH, Lee NY, Suh JS, Lee KS, et al. A pilot study of cytoreductive chemotherapy combined with infusion of additional peripheral blood stem cells reserved at time of harvest for transplantation in case of relapsed hematologic malignancies after allogeneic peripheral blood stem cell transplant. Bone Marrow Transpl. 2004; 33: 231-236.

12. Armand P, Kim HT, Logan BR, Wang Z, Alyea EP, Kalaycio ME, et al. Validation and refinement of the Disease Risk Index for allogeneic stem cell transplantation. Blood. 2014; 123: 3664-3671.

13. Grimwade D, Hills RK, Moorman AV, Walker H, Chatters S, Goldstone AH, et al. Refinement of cytogenetic classification in acute myeloid leukemia: determination of prognostic significance of rare recurring chromosomal abnormalities amongst 5876 younger adult patients treated in the United Kingdom Medical Research Council trials. Blood. 2010; 116: 354-365.

14. Greenberg P, Cox C, LeBeau MM, Fenaux P, Morel P, Sanz G, et al. International scoring system for evaluating prognosis in myelodysplastic syndromes. Blood. 1997; 89: 2079-2088.

15. Przepiorka D, Weisdorf D, Martin P, Klingemann HG, Beatty P, Hows J, et al. 1994 Consensus conference on acute GVHD grading. Bone Marrow Transplant. 1995; 15: 825-828.

16. Filipovich AH, Weisdorf D, Pavletic S, Socie G, Wingard JR, Lee SJ, et al. National Institutes of Health consensus development project on criteria for clinical trials in chronic graft-versus-host disease: I. Diagnosis and staging working group report. Biol Blood Marrow Transplant. 2005; 11: 945-956.

17. Ruggeri A, Labopin M, Ciceri F, Mohty M, Nagler A. Definition of GvHD-free, relapse-free survival for registry-based studies: an ALWP-EBMT analysis on patients with AML in remission. Bone Marrow Transplant. 2016; 51: 610-611.

18. Solh M, Zhang X, Connor K, Brown S, Solomon SR, Morris LE, et al. Postrelapse survival after haploidentical transplantation vs matched-related or matched-unrelated hematopoietic cell transplantation. Bone Marrow Transplant. 2016; 51: 949-954.

19. Kanda Y. Investigation of the freely available easy-to-use software "EZR" for medical statistics. Bone Marrow Transplant. 2013; 48: 452-458.

20. Baron F, Maris MB, Storer BE, Sandmaier BM, Panse JP, Chauncey TR, et al. High doses of transplanted CD34+ cells are associated with rapid T-cell engraftment and lessened risk of graft rejection, but not more graftversus-host disease after nonmyeloablative conditioning and unrelated hematopoietic cell transplantation. Leukemia. 2005; 19: 822-828.

21. Dhédin N, Prébet T, De Latour RP, Katsahian S, Kuentz M, Piard N, et al. Extensive chronic GVHD is associated with donor blood CD34+ cell count after G-CSF mobilization in non-myeloablative allogeneic PBSC transplantation. Bone Marrow Transplant. 2012; 47: 1564-1568.

22. Czerw T, Labopin M, Schmid C, Cornelissen JJ, Chevallier $P$, et al. High CD3 + and CD34 + peripheral blood stem cell grafts content is associated with increased risk of graft-versus-host disease without beneficial effect on disease control after reduced-intensity conditioning allogeneic transplantation from matched unrelated donors for acute myeloid leukemia-an analysis from the Acute Leukemia Working Party of the European Society for Blood and Marrow Transplantation. Oncotarget. 2016; 7: 27255-27266.

23. Rutella S, Zavala F, Danese S, Kared H, Leone G. Granulocyte colonystimulating factor: a novel mediator of $\mathrm{T}$ cell tolerance. J Immunol. 2005; 175:7085-7091.

24. Anderlini P, Champlin RE. Biologic and molecular effects of granulocyte colony-stimulating factor in healthy individuals: recent findings and current challenges. Blood. 2008; 111: 1767-1772.
Ann Hematol Oncol - Volume 6 Issue 3 - 2019 ISSN : 2375-7965 | www.austinpublishing group.com Sohn et al. @ All rights are reserved
Citation: Lee YJ, Moon JH and Sohn SK. Benefit of Granulocyte Colony-Stimulating Factor-Primed Donor Lymphocyte Infusion using Cryopreserved Cells for Patients with Acute Leukemia Who Relapsed Late after Hematopoietic Cell Transplantation. Ann Hematol Oncol. 2019; 6(3): 1236. 